\title{
Supporting Information of Meso-Scale Simulations of Quaternary Ammonium Tethered Triblock Copolymers: Effects of the Degree of Functionalization and Styrene Content
}

\author{
Xubo Luo, ${ }^{\dagger}$ Hongjun Liu, ${ }^{\dagger}$ Chulsung Bae, ${ }^{\ddagger}$ Mark E. Tuckerman, ${ }^{\S}{ }^{\sharp}$ Michael A. Hickner, ${ }^{\|}$and \\ Stephen J. Paddison*, $\dagger$ \\ $\dagger$ Department of Chemical \& Biomolecular Engineering, University of Tennessee, Knoxville, Tennessee 37996, \\ United States \\ $¥$ Department of Chemistry and Chemical Biology, Rensselaer Polytechnic Institute, Troy, New York 12180, United \\ States \\ $\S$ Department of Chemistry, New York University, New York City, New York 10003, United States \\ \# Courant Institute of Mathematical Sciences, New York University, New York, New York 10012 \\ " NYU-ECNU Center for Computational Chemistry at NYU Shanghai, 3663 Zhongshan Road North, Shanghai \\ 200062, China. \\ " Department of Materials Science and Engineering, The Pennsylvania State University, University Park, Pennsylvania \\ 16802, United States
}


Table S1. Bonded parameters in DPD simulations

\begin{tabular}{cll|ccc}
\hline Bond & $\mathrm{K}_{\mathrm{b}}$ & $\mathrm{R}_{\mathrm{b}}{ }^{0}$ & Angle & $\mathrm{K}_{\mathrm{a}}$ & $\theta_{\mathrm{a}}{ }^{0}$ \\
\hline Bs-Bs & 30 & 0.7 & Bs-Bs-Bs & 15 & 150 \\
Bs-Ph & 30 & 0.7 & Bs-Bs-Bm & 10 & 150 \\
Bs-Bm & 30 & 0.7 & Bs-Bm-Bm & 1 & 90 \\
Bm-Bm & 20 & 0.7 & Bm-Bm-Bm & 1 & 90 \\
Ph-TMM & 20 & 0.7 & Bs-Ph-TMM & 1 & 90 \\
TMM-TMA & 20 & 0.7 & Ph-TMM- TMA & 1 & 90 \\
\hline \multicolumn{2}{c}{$U^{S}=\sum_{\text {all bonds }}$} & $\frac{1}{2} K_{b}\left(r_{b}-r_{b, 0}\right)^{2}$ & \multicolumn{2}{|c}{$U^{A}=\sum_{\text {all angles }} K_{a}\left(1-\cos \left(\theta_{a}-\theta_{a, 0}\right)\right)$} \\
\hline
\end{tabular}

Table S2. Non-bonded parameters in DPD simulations

\begin{tabular}{c|cccccc}
\hline$a_{\mathrm{ij}}$ & Bs, Bm & Ph & TMM & TMA $^{+}$ & $\mathrm{OH}^{-}$ & W \\
\hline $\mathrm{Bs}, \mathrm{Bm}$ & 30.28 & 35.02 & 29.24 & 27.52 & 34.80 & 35.57 \\
$\mathrm{Ph}$ & 35.02 & 30.53 & 27.74 & 27.31 & 30.05 & 33.44 \\
$\mathrm{TMM}$ & 29.24 & 27.74 & 26.03 & 25.59 & 27.88 & 27.46 \\
$\mathrm{TMA}^{+}$ & 27.52 & 27.31 & 25.59 & 65.79 & -15.02 & 26.61 \\
$\mathrm{OH}^{-}$ & 34.80 & 30.05 & 27.88 & -15.02 & 65.68 & 27.34 \\
$\mathrm{~W}$ & 35.57 & 33.44 & 27.46 & 26.61 & 27.34 & 25.01 \\
\hline
\end{tabular}


Table S3. Equivalent IEC for the simulated systems

\begin{tabular}{|c|c|c|}
\hline $\mathrm{DF}$ & Sty $\%$ & IEC/ [mequiv/g] \\
\hline $25 \%$ & \multirow{3}{*}{$17 \mathrm{~mol} \%$} & 0.62 \\
\hline $50 \%$ & & 1.15 \\
\hline $75 \%$ & & 1.60 \\
\hline \multirow{2}{*}{$50 \%$} & $10 \mathrm{~mol} \%$ & 0.74 \\
\hline & $42 \mathrm{~mol} \%$ & 2.03 \\
\hline
\end{tabular}

Table S4. Equivalent water uptake (WU) for the simulated systems

\begin{tabular}{|c|c|c|c|c|c|c|}
\hline \multirow{2}{*}{$\mathrm{DF}$} & \multirow{2}{*}{ Sty $\% \lambda$} & \multicolumn{5}{|c|}{ WU\% } \\
\hline & & 4 & 8 & 12 & 16 & 20 \\
\hline $25 \%$ & \multirow{3}{*}{$17 \mathrm{~mol} \%$} & 4.5 & 8.9 & 13.4 & 17.9 & 22.3 \\
\hline $50 \%$ & & 8.3 & 16.6 & 24.8 & 33.1 & 41.4 \\
\hline $75 \%$ & & 11.6 & 23.1 & 34.6 & 46.2 & 57.7 \\
\hline \multirow{2}{*}{$50 \%$} & $10 \mathrm{~mol} \%$ & 5.9 & 11.8 & 17.8 & 23.7 & 29.6 \\
\hline & $42 \mathrm{~mol} \%$ & 14.6 & 29.3 & 43.9 & 58.5 & 73.2 \\
\hline
\end{tabular}




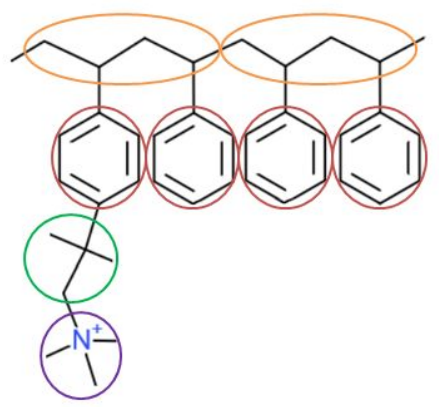

(a)

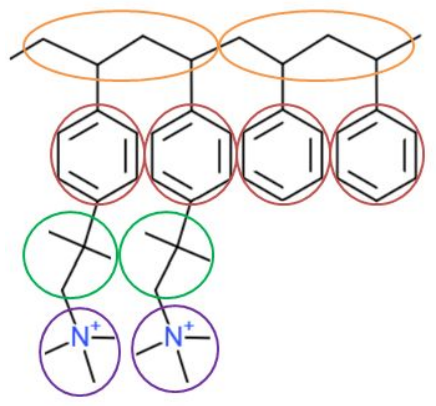

(c)

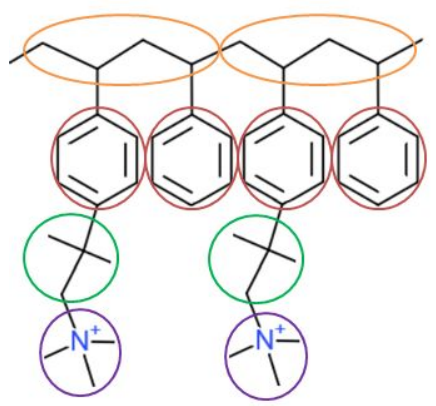

(b)

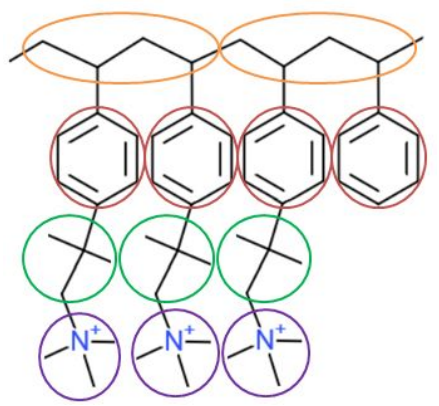

(d)

Figure S1. Atomic topology of the polystyrene block with different degrees of functionalization (DF). (a) $25 \%$; (b) $50 \%$; (c) $50 \%$ alternative; and (d) $75 \%$. Only one repeat unit is shown for each DF. 


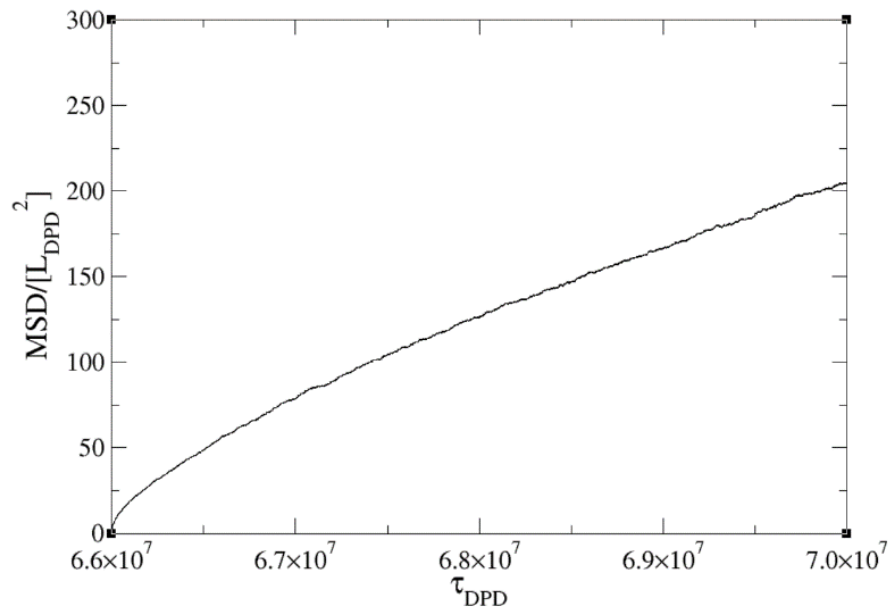

Figure S2. Mean square displacement of $\mathrm{Ph}$ beads for SEBS with $\mathrm{Sty} \%=17 \mathrm{~mol} \%$ and $\mathrm{DF}=50 \%$ alternative at $\lambda=12$. The MSD is more than 200 squared DPD length units in 4 million steps. For the whole simulation length, it is about 247 DPD length units, which is more than four times of the simulation box. 


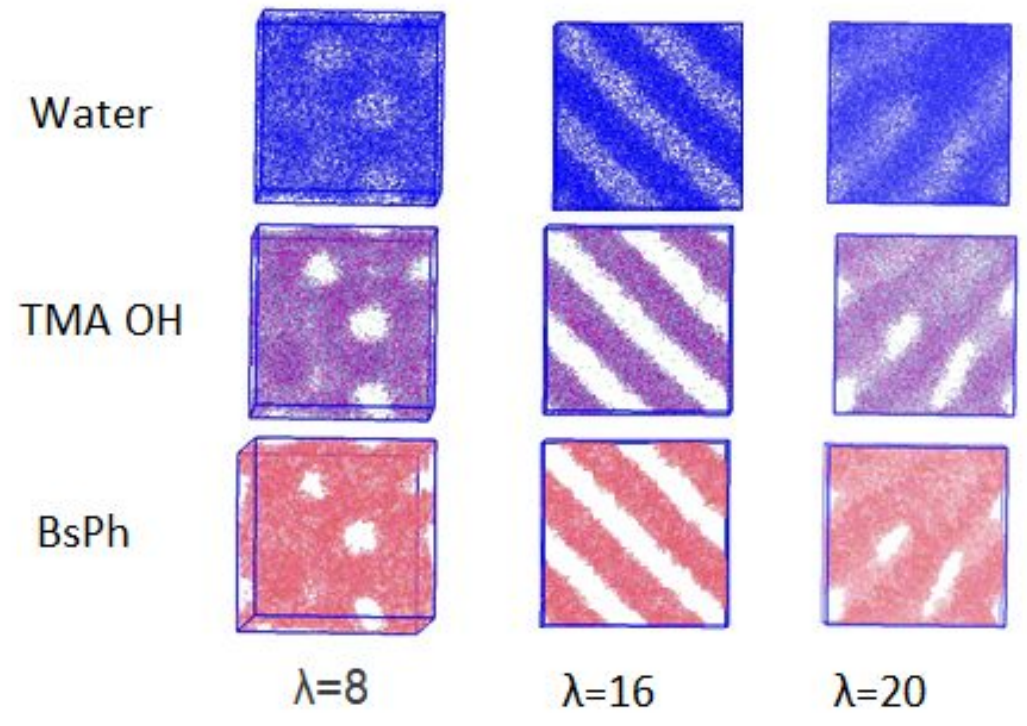

Figure S3. Water distribution of 50\% DF and $17 \mathrm{~mol} \%$ of polystyrene. Most of the water beads are in the hydrophilic phase which consists of both polystyrene and functional groups. There is always a small amount of water scattered in the hydrophobic phase. 


\begin{tabular}{|l|l|l|l|l|l|}
\hline$\lambda$ & $\mathrm{DF}$ & \multicolumn{2}{c|}{ Slice } & $\mathrm{BsPh}$ \\
\hline 8 & & & & & \\
\hline
\end{tabular}

Figure S4. Morphologies of imperfect lamella. All rows are of the SEBS with $17 \mathrm{~mol} \%$ of polystyrene with varying degree of functionalization (DF). In each sub domain, the morphology is perfect lamella, but the whole simulation box does not show the lamella structure. Color scheme: Orange: Bs and Bm, Mauve: Ph, Green: TMM, Purple: TMA, Cyan: OH, Blue: Water. 


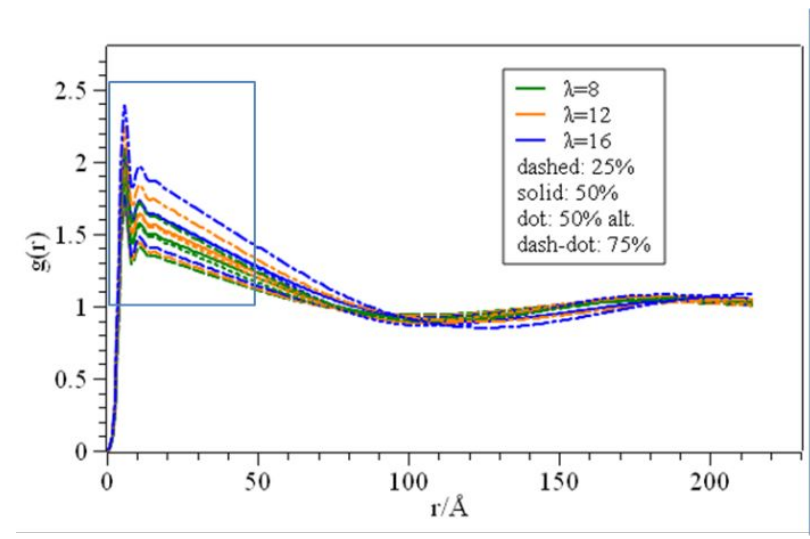

(a)

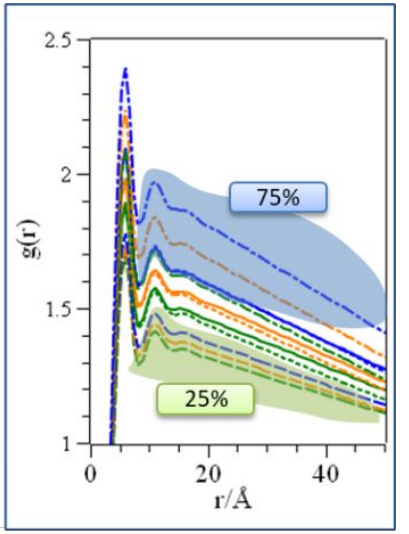

(b)

Figure S5. Radial distribution function of the polyethylene mid-block for different DFs. The coordinates of Bm beads are used for calculation. (a) is the plot out to $210 \AA$; and (b) is a zoom-in view of the second peak. Higher DF results in higher peaks, which indicates that the functional side chains lead to concentration and structuring of the polyethylene beads. 

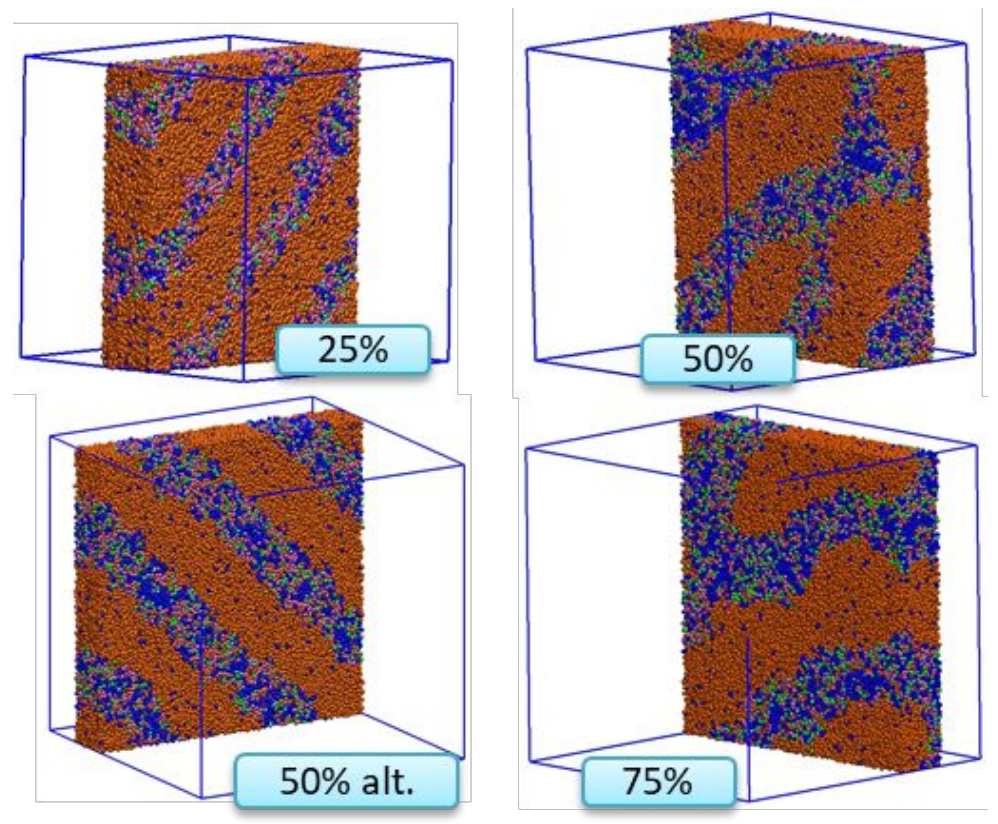

(a)
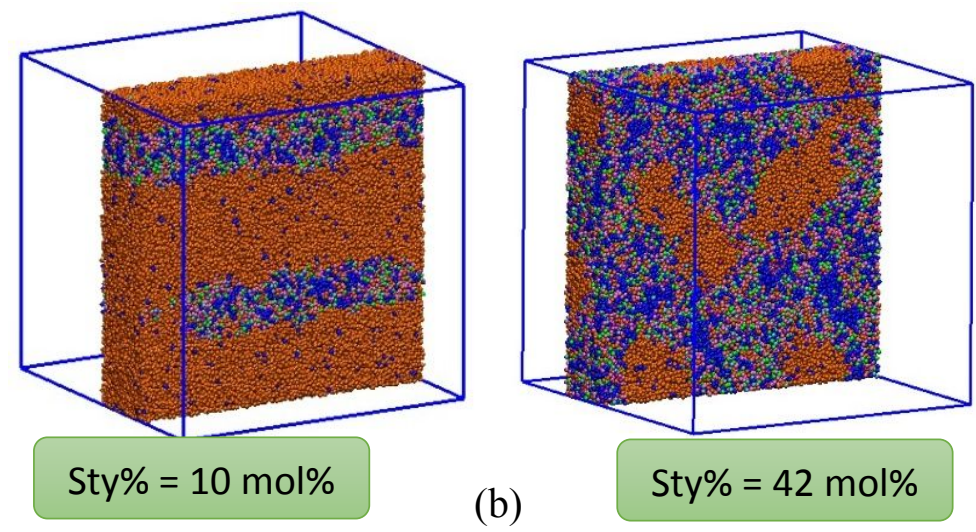

Figure S6. Slices of the hydrated morphology at the hydration level of 16. (a) Different DF with $17 \mathrm{~mol} \%$ of polystyrene in SEBS. (b) $50 \%$ DF with 10 and $42 \mathrm{~mol} \%$ of polystyrene in SEBS. Water-only domains of dark blue clusters are observed for all cases except that the $25 \%$ DF in (a) does not obviously show this phenomenon. 


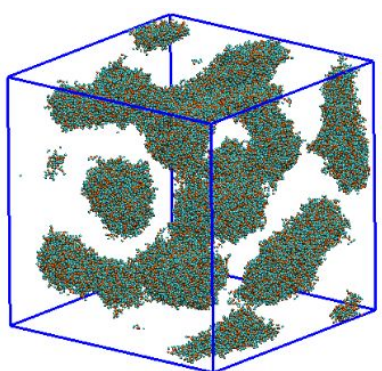

(a)

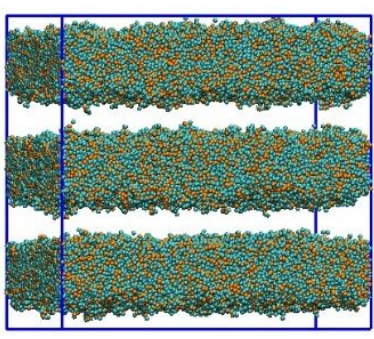

(b)

Figure S7. Simulated morphology of pristine SEBS. Only the polystyrene end-blocks, i.e., the Bs(orange) and $\mathrm{Ph}$ (cyan) beads, are shown for clarity. (a) and (b) are Sty\% $=10 \mathrm{~mol} \%$ and $42 \mathrm{~mol} \%$, respectively. 


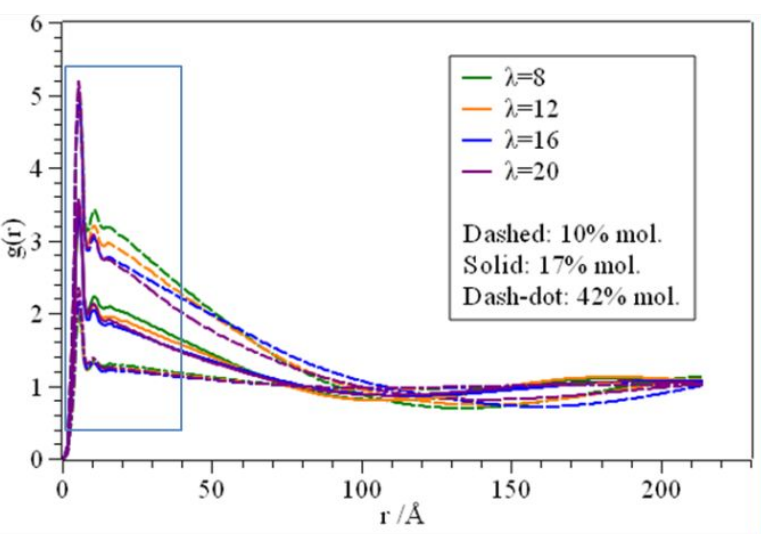

(a)

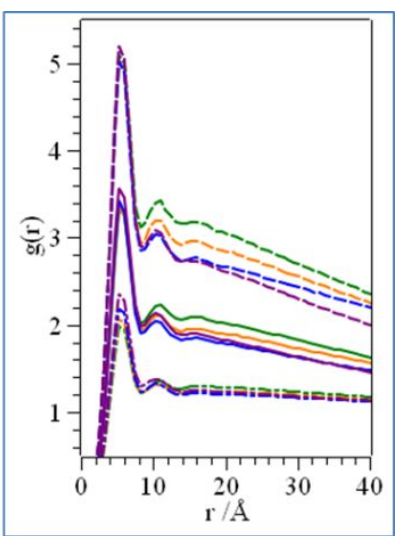

(b)

Figure S8. Comparison of radial distribution function of polystyrene block of 10, 17 and $42 \mathrm{~mol} \%$ of polystyrene in SEBS. At $10 \mathrm{~mol} \%$ the peaks are higher for all the five hydration levels, while at $42 \mathrm{~mol} \%$ shows almost flat curves after the first peak. 


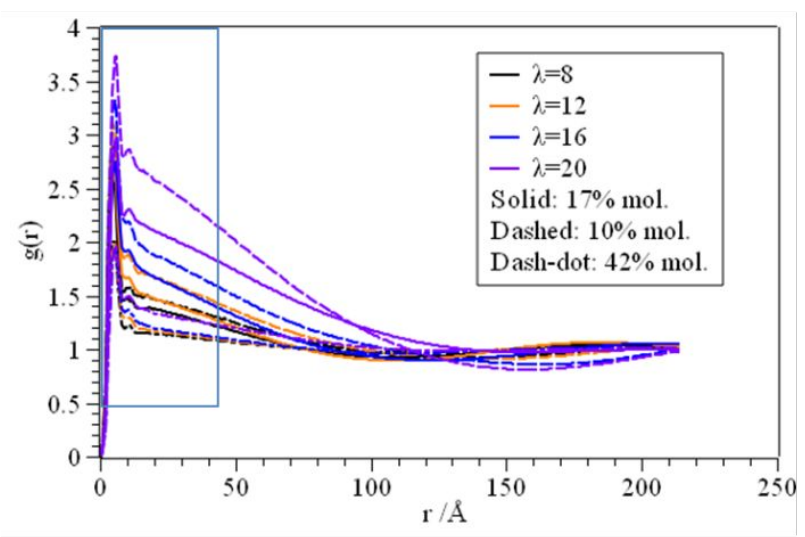

(a)

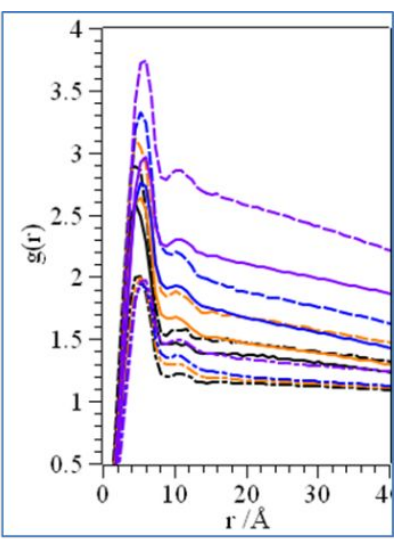

(b)

Figure S9. Comparison of the radial distribution function of water of 10,17 and $42 \mathrm{~mol} \%$ of polystyrene in SEBS. Sty $\%=10 \mathrm{~mol} \%$ gives higher peaks for all five hydration levels, while Sty $\%=42$ displays very low peaks. 


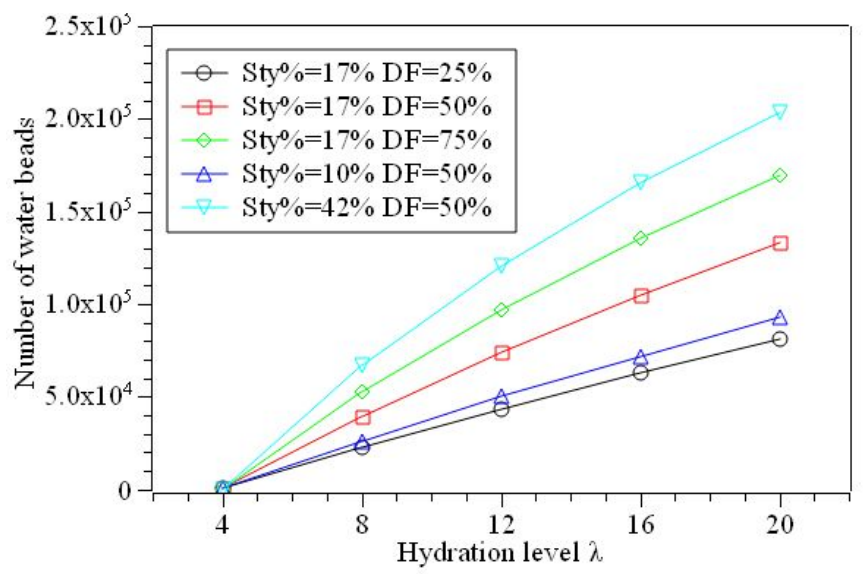

Figure S10. Comparison of the total number of water beads in the simulation box. The definition of hydration level determines that even at the same hydration level, the amount of water in the simulation box is different. The morphology shows that most of the water beads are in the hydrophilic phase of polystyrene and functional groups, the definition of hydration level was kept as the number of $\mathrm{H}_{2} \mathrm{O}$ per fixed ionic site. 